University of Nebraska - Lincoln

DigitalCommons@University of Nebraska - Lincoln

\title{
Combined finite element and peridynamic analyses for predicting failure in a stiffened composite curved panel with a central slot
}

\author{
Erkan Oterkus \\ The University of Arizona, Tucson, AZ \\ Erdogan Madenci \\ The University of Arizona, Tucson, AZ \\ Olaf Weckner \\ Boeing Research \& Technology, Seattle, WA \\ Stewart Silling \\ Sandia National Laboratories, Albuquerque, NM \\ Philip Bogert \\ NASA Langley Research Center, Hampton, VA \\ See next page for additional authors
}

Follow this and additional works at: https://digitalcommons.unl.edu/usdoepub

Part of the Bioresource and Agricultural Engineering Commons

Oterkus, Erkan; Madenci, Erdogan; Weckner, Olaf; Silling, Stewart; Bogert, Philip; and Tessler, Alexander, "Combined finite element and peridynamic analyses for predicting failure in a stiffened composite curved panel with a central slot" (2012). US Department of Energy Publications. 127.

https://digitalcommons.unl.edu/usdoepub/127

This Article is brought to you for free and open access by the U.S. Department of Energy at DigitalCommons@University of Nebraska - Lincoln. It has been accepted for inclusion in US Department of Energy Publications by an authorized administrator of DigitalCommons@University of Nebraska - Lincoln. 


\section{Authors}

Erkan Oterkus, Erdogan Madenci, Olaf Weckner, Stewart Silling, Philip Bogert, and Alexander Tessler 


\title{
Combined finite element and peridynamic analyses for predicting failure in a stiffened composite curved panel with a central slot
}

\author{
Erkan Oterkus ${ }^{\mathrm{a}}$, Erdogan Madenci ${ }^{\mathrm{a}, *}$, Olaf Weckner ${ }^{\mathrm{b}}$, Stewart Silling ${ }^{\mathrm{c}}$, Philip Bogert ${ }^{\mathrm{d}}$, Alexander Tessler $^{\mathrm{d}}$ \\ a Department of Aerospace and Mechanical Engineering, The University of Arizona, Tucson, AZ 85721-0119, United States \\ ${ }^{\mathrm{b}}$ Boeing Research E' Technology, Seattle, WA 98124-2207, United States \\ c Multiscale Dynamic Material Modeling Department, Sandia National Laboratories, Albuquerque, NM 87185-1322, United States \\ d Structural Mechanical and Concepts Branch, NASA Langley Research Center, Hampton, VA 23681-2199, United States
}

\section{A R T I C L E I N F O}

\section{Article history:}

Available online 25 August 2011

\section{Keywords:}

Progressive

Failure

Composites

Nonlocal

Peridynamic theory

\begin{abstract}
A B S T R A C T
This study presents an analysis approach based on a merger of the finite element method and the peridynamic theory. Its validity is established through qualitative and quantitative comparisons against the test results for a stiffened composite curved panel with a central slot under combined internal pressure and axial tension. The predicted initial and final failure loads, as well as the final failure modes, are in close agreement with the experimental observations. This approach demonstrates the capability of the PD approach to assess the durability of complex composite structures.
\end{abstract}

(c) 2011 Elsevier Ltd. All rights reserved.

\section{Introduction}

Despite the development of many important concepts to predict material behavior and failure, the prediction of failure modes and residual strength of composite materials is a challenge within the framework of the finite element method (FEM). Furthermore, the previous methods cannot address the nucleation of damage in a continuous material. The field of fracture mechanics is primarily concerned with the evolution of pre-existing defects within a body, rather than the nucleation of new defects. Even when addressing crack growth, the FEM suffers from the inherent limitation that it requires remeshing after each incremental crack growth. In addition to the need to remesh, existing methods for fracture modeling also suffer from the requirement of an external crack growth criterion. This criterion prescribes how damage evolves a priori based on local conditions, and guides the analysis as to when and how damage initiates and propagates. Considering the difficulty in obtaining and generalizing experimental fracture data, providing such a criterion for damage growth, especially in composite structures, clearly presents a major obstacle to fracture modeling using conventional methods. This prevents such methods from being applicable to problems in which multiple damage growth occurs and interacts in complex patterns.

\footnotetext{
* Corresponding author. Fax: +1 5206218191.

E-mail addresses: oterkus@email.arizona.edu (E. Oterkus), madenci@email. arizona.edu (E. Madenci), Olaf.Weckner@boeing.com (O. Weckner), sasilli@sandia. gov (S. Silling), philip.b.bogert@nasa.gov (P. Bogert), alexander.tessler-1@nasa.gov (A. Tessler).
}

The governing equations of the FEM are based on the partial differential equations (PDEs) of classical continuum mechanics and that the spatial derivatives required by the PDEs do not, by definition, exist at crack tips or along crack surfaces. Therefore, the basic mathematical structure of the formulation breaks down whenever a crack appears in a body. Various special techniques have been developed in fracture mechanics to deal with this limitation. Generally, these techniques involve redefining a body in such a way as to exclude the crack, then applying conditions at the crack surfaces as boundary conditions.

In order to overcome these problems, Silling [1] introduced a nonlocal theory that does not require spatial derivatives-the peridynamic (PD) theory. The main difference between the PD theory and classical continuum mechanics is that the former is formulated using integral equations as opposed to derivatives of the displacement components. This feature allows damage initiation and propagation at multiple sites, with arbitrary paths inside the material, without resorting to special crack growth criteria. In the PD theory, internal forces are expressed through nonlocal interactions between the material points within a continuous body, and damage is a part of the constitutive model. Interfaces between dissimilar materials have their own properties and damage can propagate when and where it is energetically favorable for it to do so.

With the PD theory, damage in the material is simulated in a much more realistic manner compared to the classical continuumbased methods. The broken interactions may align themselves along surfaces that form cracks and the deformation is discontinuous across such as a crack, yet the integral equations continue to remain valid. The PD theory has been utilized successfully for damage prediction in many problems. 
The PD theory was applied successfully by Askari et al. [2] and Colavito et al. [3,4] to predict damage in laminated composites subjected to low-velocity impact and damage in woven composites subjected to static indentation. Furthermore, Xu et al. [5] considered notched laminated composites under biaxial loads. Recently, Kilic et al. [6] predicted the basic failure modes of fiber, matrix, and delamination in various laminates with a pre-existing central crack under tension. Also, Oterkus et al. [7] demonstrated that PD analysis is capable of capturing bearing and shear-out failure modes in bolted composite lap-joints.

This study presents a coupling of the FEM with PD theory through a submodeling approach to investigate damage in complex composite structures by considering a stiffened composite curved panel with a central slot (NASA Panel 67) subjected to combined internal pressure and axial tension loading. The initial and final failure loads of the panel and failure modes due to the applied loading are evaluated by using PD theory and compared against test measurements and observations.

\section{Peridynamic theory}

The deformation response of solid structures subjected to external forces can be obtained by assuming the structure to be a continuous body or a continuum, without paying attention to its atomistic structure. Hence, it is possible to perform both static and dynamic analyses of large structures within a reasonable amount of time. The conventional approach that is used to analyze solid structures is known as "classical continuum mechanics" and has been successfully applied to numerous problems in the past. Within the classical continuum mechanics framework, it is assumed that the continuous body is composed of an infinite number of infinitesimal volumes, which are called material points. These material points interact with each other only if they are within the nearest neighborhood of each other. These interactions are expressed in terms of contact forces or tractions, $\mathbf{T}$, as shown in Fig. 1.

Using the conservation of linear momentum and relating the traction vectors, $\mathbf{T}$, to the well-known stress tensor, $\boldsymbol{\sigma}$, results in the equation of motion of the material point, $\mathbf{x}$, in classical continuum mechanics

$$
\rho(\mathbf{x}) \ddot{\mathbf{u}}(\mathbf{x}, t)=\nabla \cdot \boldsymbol{\sigma}+\mathbf{b}(\mathbf{x}, t)
$$

where $\rho(\mathbf{x}), \mathbf{b}(\mathbf{x}, t)$, and $\ddot{\mathbf{u}}(\mathbf{x}, t)$ represent the mass density, body force density, and acceleration, respectively, of the material point located at $\mathbf{x}$. The spatial derivatives in the divergence operation associated with the stress tensor, $\boldsymbol{\sigma}$, do not exist on the discontinuity in the structure. Therefore, Eq. (1) is not valid for problems including discontinuities, such as cracks. Silling [1] replaced the divergence term in Eq. (1) with an integral term, which makes the new form of the equation of motion applicable whether or not a discontinuity exists in the structure:

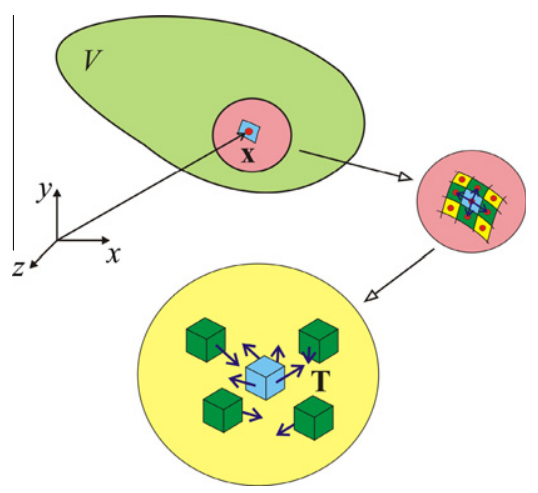

Fig. 1. Interaction of material points in classical continuum mechanics. $\rho(\mathbf{x}) \ddot{\mathbf{u}}(\mathbf{x}, t)=\int_{H} \mathbf{f}\left(\mathbf{x}^{\prime}-\mathbf{x}, \mathbf{u}^{\prime}-\mathbf{u}\right) d H+\mathbf{b}(\mathbf{x}, t)$

In Eq. (2), the horizon, $H$, includes all the material points that the material point $\mathbf{x}$ can interact with inside the body. The interaction force or peridynamic force between material points $\mathbf{x}$ and $\mathbf{x}^{\prime}$ can be expressed as $\mathbf{f}\left(\mathbf{x}^{\prime}-\mathbf{x}, \mathbf{u}^{\prime}-\mathbf{u}\right)$, and it is a function of the relative position vector, $\mathbf{x}^{\prime}-\mathbf{x}$, and relative displacement vector, $\mathbf{u}^{\prime}-\mathbf{u}$. The peridynamic force is along the same direction of the relative position of these material points in the deformed configuration, i.e., $\mathbf{y}^{\prime}-\mathbf{y}=\left(\mathbf{x}^{\prime}+\mathbf{u}^{\prime}\right)-(\mathbf{x}+\mathbf{u})$. For an elastic isotropic material, the peridynamic force takes the form

$\mathbf{f}=\operatorname{cs} \frac{\mathbf{y}^{\prime}-\mathbf{y}}{\left|\mathbf{y}^{\prime}-\mathbf{y}\right|}$

where $c$ and $s$ represent the peridynamic material parameter and stretch between material points $\mathbf{x}$ and $\mathbf{x}^{\prime}$, respectively. The stretch, $s$, is defined as

$S=\frac{\left|\mathbf{y}^{\prime}-\mathbf{y}\right|-\left|\mathbf{x}^{\prime}-\mathbf{x}\right|}{\left|\mathbf{X}^{\prime}-\mathbf{X}\right|}$

The material parameter, $c$, can be related to the engineering material constants by equating the strain energy densities of the PD and classical continuum theories at a material point inside a body due to simple loading such as uniform expansion. Silling and Askari [8] derived an explicit expression for parameter $c$ in the form

$c=\frac{18 \kappa}{\pi \delta^{4}}$

where $\kappa$ is the bulk modulus of the material and $\delta$ represents the radius of a spherical horizon.

It can also be assumed that two material points, $\mathbf{x}$ and $\mathbf{x}^{\prime}$, cease to interact with each other if the stretch between these material points exceeds a critical stretch value, $s_{0}$, as shown in Fig. 2 . This material model represents an elastic material behavior without allowing any permanent deformation.

Termination of the interaction between material points can be associated with the failure of the material by modifying the peridynamic force relation given in Eq. (3) by introducing the failure parameter $\mu\left(\mathbf{x}^{\prime}-\mathbf{x}, t\right)$

$\mathbf{f}=\mu\left(\mathbf{x}^{\prime}-\mathbf{x}, t\right) \operatorname{cs} \frac{\mathbf{y}^{\prime}-\mathbf{y}}{\left|\mathbf{y}^{\prime}-\mathbf{y}\right|}$

where the failure parameter can be defined as

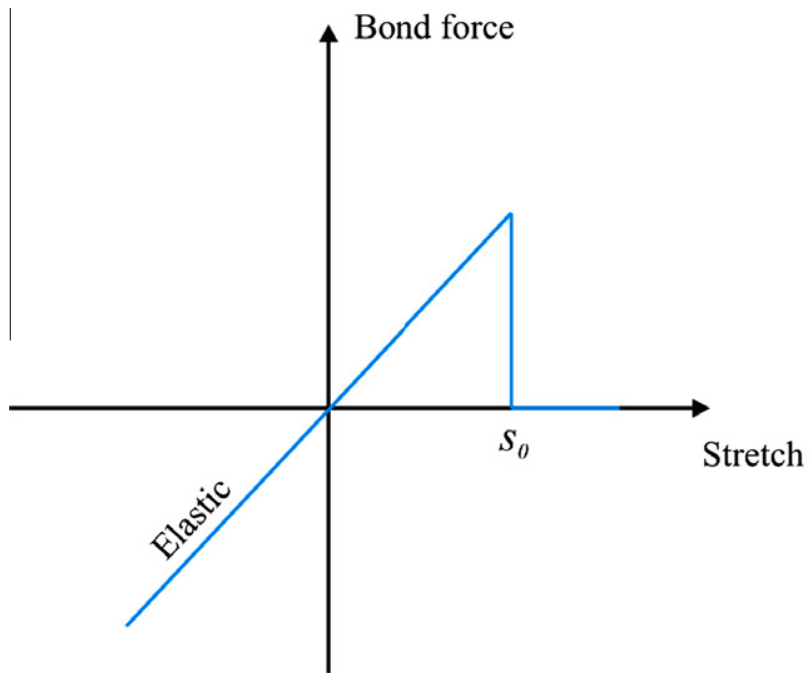

Fig. 2. Constitutive relation between material points in an elastic material. 


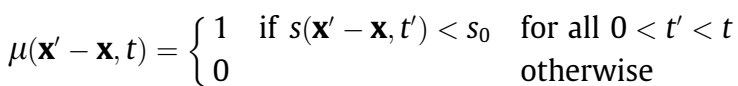

The inexplicit nature of local damage at a material point, $\mathbf{x}$, arising from the introduction of failure in the constitutive model, is removed by defining the local damage as

$\varphi(\mathbf{x}, t)=1-\frac{\int_{H} \mu\left(\mathbf{x}^{\prime}-\mathbf{x}, t\right) d H}{\int_{H} d H}$

Thus, local damage is the weighted ratio of the number of the broken interactions to the total number of interactions within the hori$z o n, H$. The extent of damage is defined by a value between 0 and 1 , where 0 indicates that a material point has no damage and 1 indicates complete damage at the material point. Also, a damage value of 0.5 and above indicates possible cracking.

In the case of isotropic materials, the critical stretch, $s_{0}$, value can be related to the equivalent energy release rate as derived by Silling and Askari [8]

$s_{0}=\sqrt{\frac{5 G_{0}}{9 \kappa \delta}}$

where $G_{0}$ is the critical energy release rate of the material and can be related to the fracture toughness of the material.

In order to solve Eq. (2), a collocation method is adopted and the numerical treatment involves the discretization of the domain of interest into subdomains. The domain can be discretized into square subdomains. With this discretization, the volume integration in Eq. (2) is approximated, leading to

$$
\begin{aligned}
\rho\left(\mathbf{x}_{(i)}\right) \ddot{\mathbf{u}}\left(\mathbf{x}_{(i)}, t\right)= & \sum_{j=1}^{N} \mathbf{f}\left(\mathbf{u}\left(\mathbf{x}_{(j)}, t\right)-\mathbf{u}\left(\mathbf{x}_{(i)}, t\right), \mathbf{x}_{(j)}-\mathbf{x}_{(i)}\right) V_{(j)} \\
& +\mathbf{b}\left(\mathbf{x}_{(i)}, t\right)
\end{aligned}
$$

where $\mathbf{x}_{(i)}$ is the position vector located at the ith collocation (material) point and $N$ is the number of subdomains within the horizon of the $i$ th material point. The position vector $\mathbf{x}_{(j)}$ represents the location of the $j$ th collocation point. The volume of the $j$ th subdomain is $V_{(j)}$. The details of the computational considerations and schemes are given by Oterkus [9].

The velocity and displacement at the next time step can be obtained by employing explicit forward and backward difference techniques in two steps, respectively. Although this explicit time integration scheme is straightforward, it is only conditionally stable. Therefore, a stability condition is necessary to obtain convergent results. A stability condition derived by Silling and Askari [8] can be used to determine the time step size, $\Delta t$ as

$$
\Delta t<\sqrt{\frac{2 \rho\left(\mathbf{x}_{(i)}\right)}{\sum_{j=1}^{M} \frac{c}{\left|\mathbf{x}_{(j)}-\mathbf{x}_{(i)}\right|} V_{(j)}}}
$$

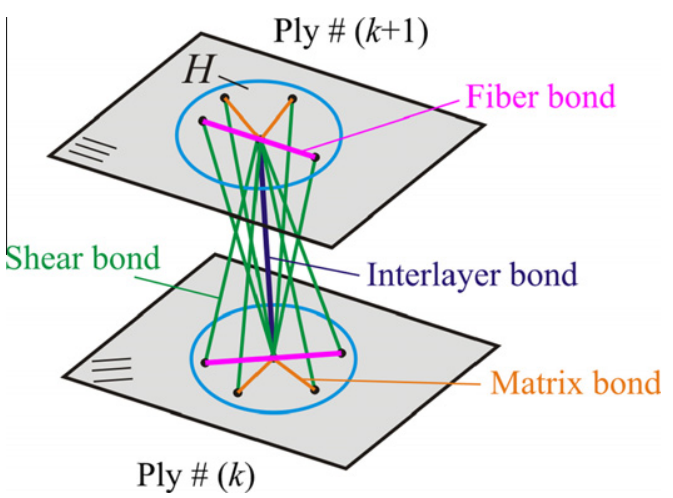

Fig. 3. Peridynamic bonds for a fiber-reinforced composite laminate.

\section{Peridynamic modeling of composite materials}

The peridynamic formulation described in the previous section concerns an isotropic material where there is no directional dependence of the interactions between the material points. However, if anisotropic materials such as a fiber-reinforced composite structure are considered, the directional dependency must be included in the peridynamic analysis. Therefore, four different peridynamic material parameters are introduced, as shown in Fig. 3, to model a fiber-reinforced composite laminate. Note that a material point of interest can only interact with a material point located either in the same ply or in the adjacent plies.

Associated with a lamina (ply), the material parameter concerning the interaction of material points in the fiber direction only is

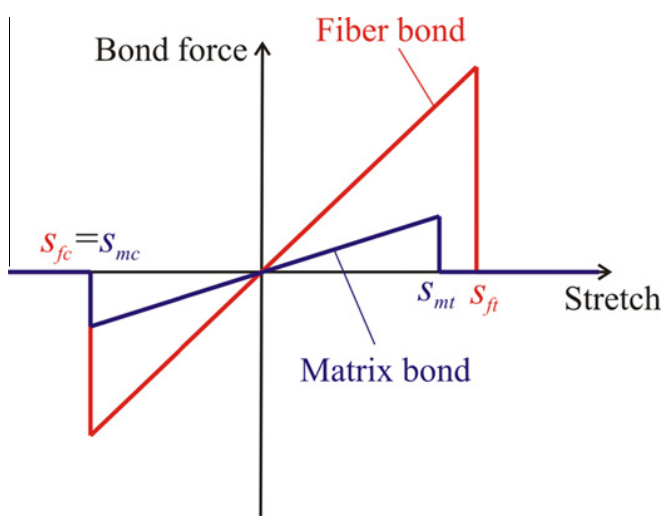

Fig. 4. Force-stretch relation for fiber and matrix bonds.

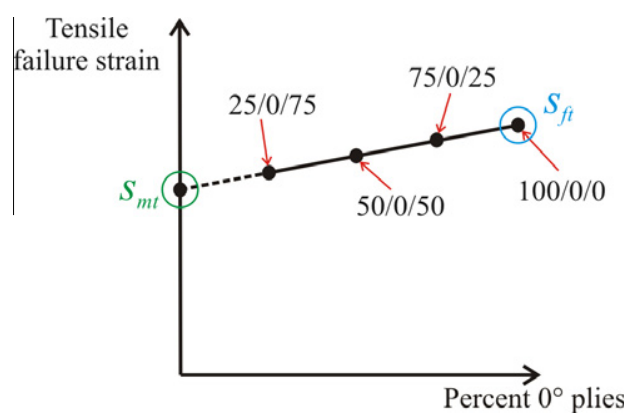

Fig. 5. Determination of critical stretch values $s_{f t}$ and $s_{m t}$.

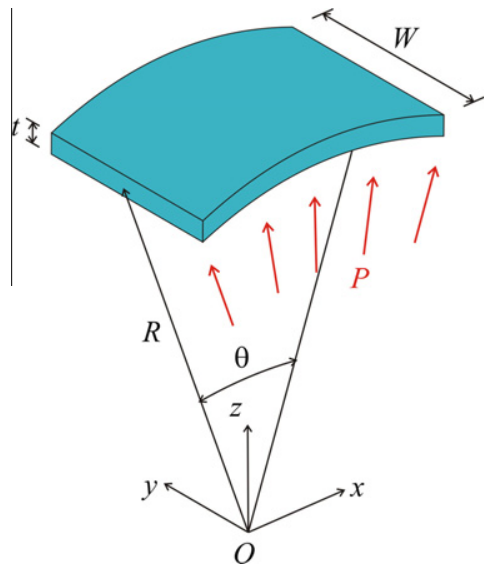

Fig. 6. Cylindrical composite shell under internal pressure. 
denoted with $c_{f}$. The interaction of material points in all other directions within a ply is governed by the material parameter $c_{m}$. The interaction between material points in adjacent plies is described by the material parameter $c_{i n}$ and $c_{i s}$ accounting for transverse normal and shear deformations between the neighboring plies.

As in the case of isotropic materials, the peridynamic material parameters, $c_{f}, c_{m}, c_{i n}$ and $c_{i s}$ can be expressed analytically in terms of the engineering material constants, $E_{1}, E_{2}, E_{3}, G_{12}$, and $v_{12}$, as

$c_{f}=\frac{2 E_{1}\left(E_{1}-E_{2}\right)}{\left(E_{1}-\frac{1}{9} E_{2}\right)\left(\sum_{q=1}^{N} \xi_{q i} V_{q}\right)}$

$$
\begin{aligned}
c_{m} & =\frac{8 E_{1} E_{2}}{\left(E_{1}-\frac{1}{9} E_{2}\right) \pi t \delta^{3}} \\
c_{i n} & =\frac{E_{m}}{t \bar{V}}
\end{aligned}
$$

and

$c_{i s}=\frac{2 G_{m}}{\pi t} \frac{1}{\left(\delta^{2}+t^{2} \ln \left(\frac{t^{2}}{\delta^{2}+t^{2}}\right)\right)}$

where $E_{m}$ and $G_{m}$ are the elastic modulus and shear modulus of the matrix material, respectively, and $\bar{V}$ is the volume of a material

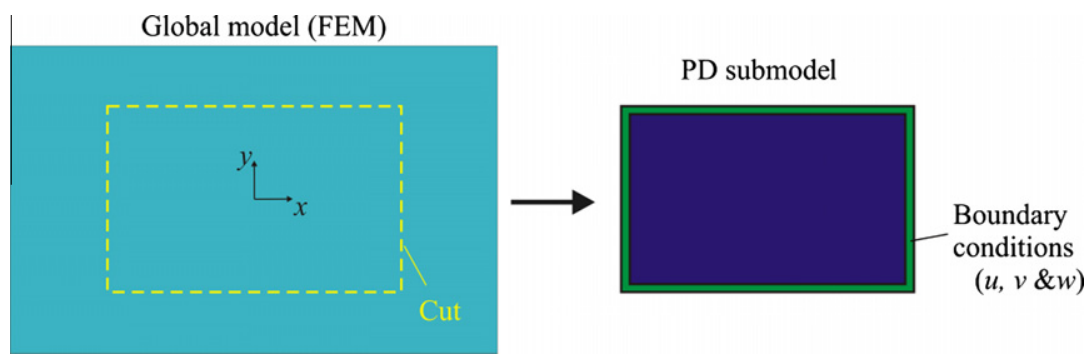

Fig. 7. Coupled FEM and PD analyses of a laminate: global FE model under internal pressure loading and PD submodel with displacement boundary conditions.

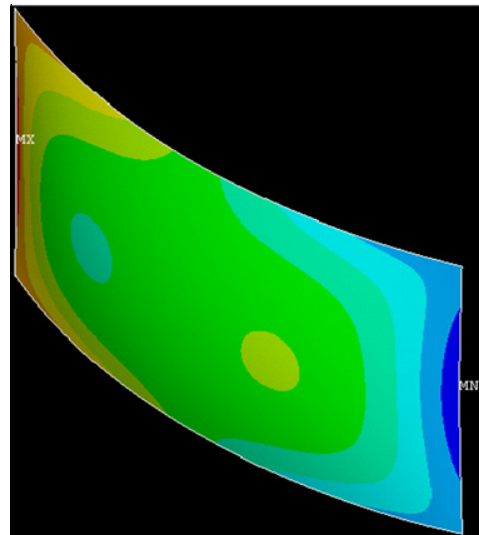

(a)

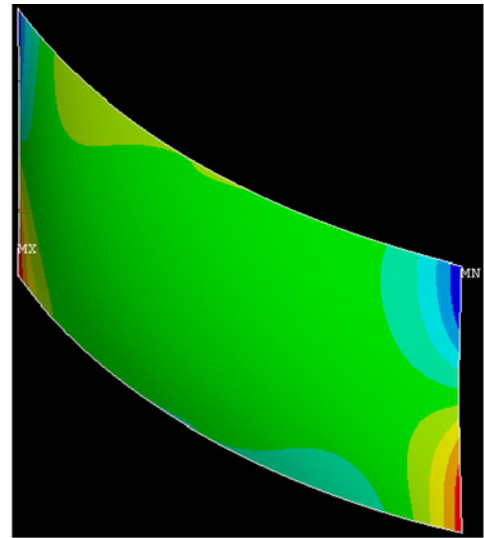

(b)

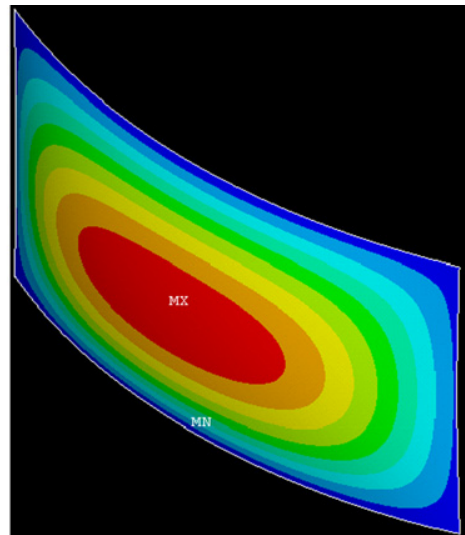

(c)

Fig. 8. FEM predictions of displacement contours: (a) $u_{0}(x, y)$, (b) $v_{0}(x, y)$, and (c) $w_{0}(x, y)$.

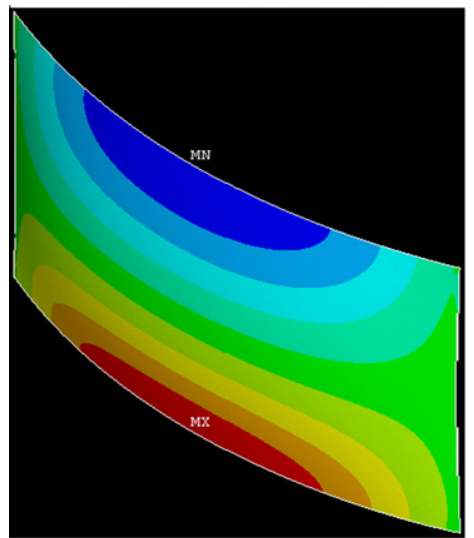

(a)

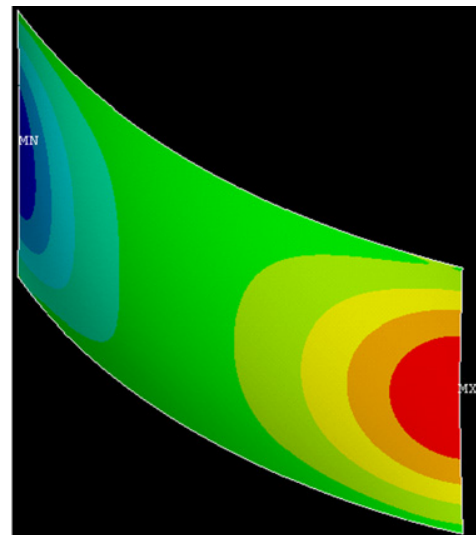

(b)

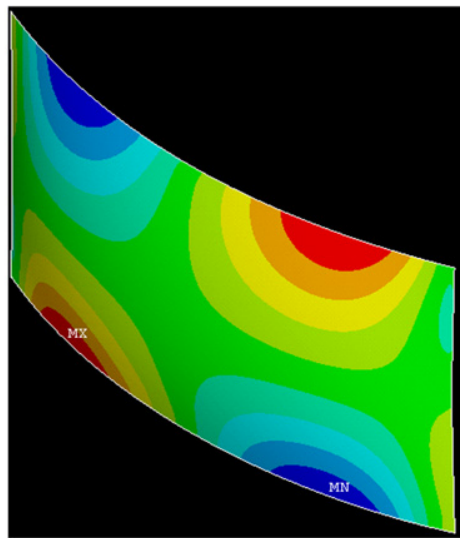

(c)

Fig. 9. FEM predictions of rotation contours: (a) $\theta_{x}(x, y)$, (b) $\theta_{y}(x, y)$, and (c) $\theta_{z}(x, y)$. 
point. The initial length of the bond in the fiber direction and its stretch after deformation between material points $q$ and $i$ are denoted by $\xi_{q i}$ and $s_{q i}$, respectively. The volume of the material point, $q$ that interacts with material point, $i$ is denoted by $V_{q}$ which can be approximated as

$V_{q}=\frac{\pi t \delta^{2}}{N}$

in which $N$ is the number of material points within its horizon, $\delta$ and $t$ is the thickness of the lamina. The detailed derivations of these expressions are given by Oterkus [9].

Because of the pair-wise interaction of the material points, four independent material constants of a lamina reduce to two independent constants along with constraints on material constants, $G_{12}$ and $v_{12}$ as

$G_{12}=\frac{v_{12} E_{2}}{1-v_{21} v_{12}}=\frac{E_{1} E_{2}}{3\left(E_{1}-\frac{1}{9} E_{2}\right)}$

and

$v_{12}=\frac{1}{3}$

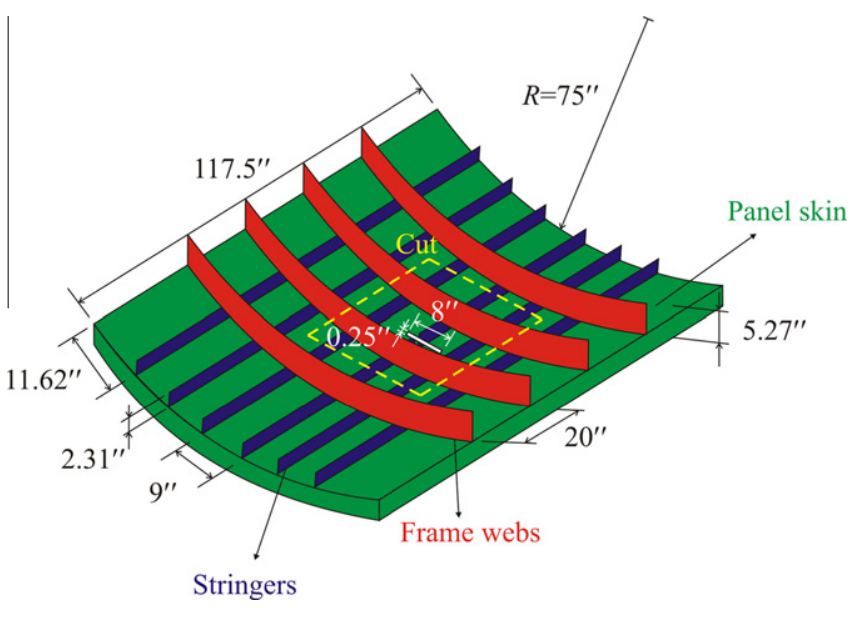

Fig. 12. Configured stiffened composite curved panel with a slot.

The detailed derivation of the limitation on $G_{12}$ and $v_{12}$ are also given by Oterkus [9].

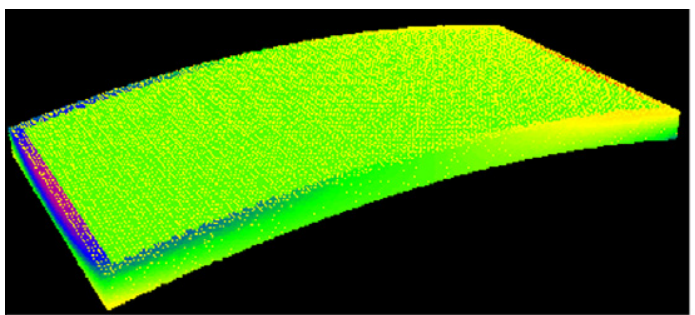

(a)

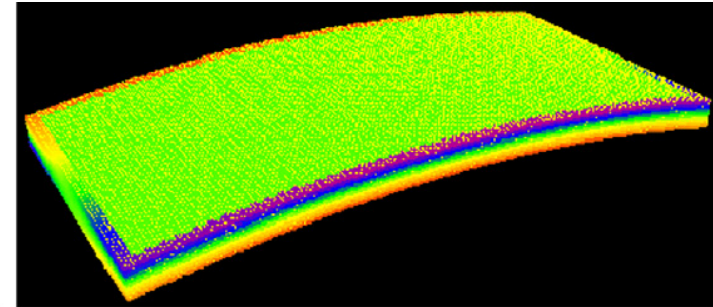

(b)

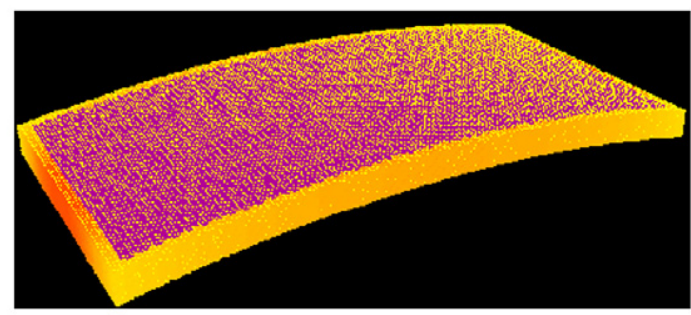

(c)

Fig. 10. Cut boundary displacements of a three-dimensional PD model: (a) $u(x, y, z),(\mathrm{b}) v(x, y, z)$, and (c) $w(x, y, z)$.

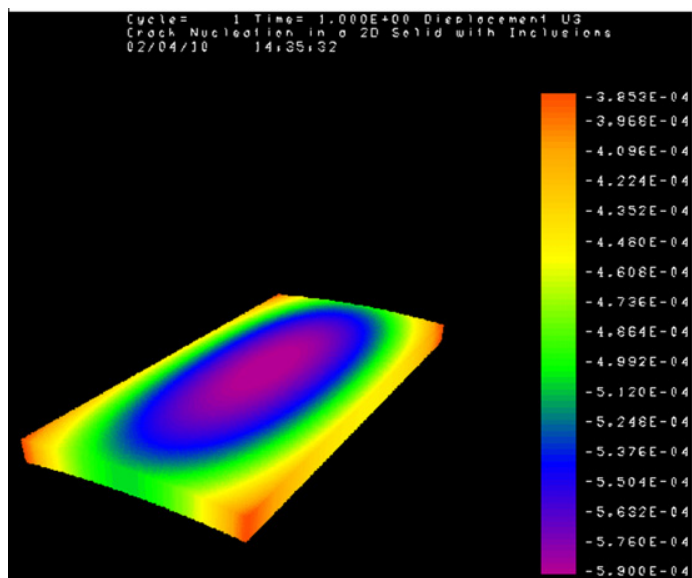

(a)

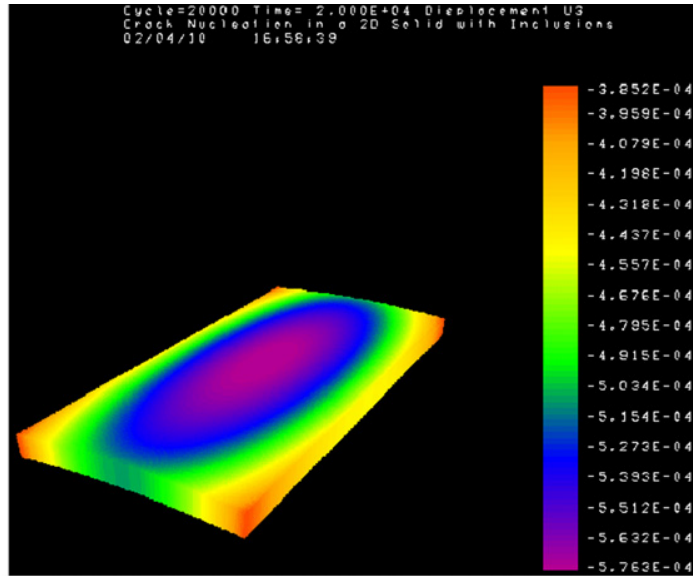

(b)

Fig. 11. Comparison of out-of-plane deflections in the submodeling region: (a) FE analysis and (b) PD analysis. 
The constitutive or the force-stretch relations for the in-plane interactions of two material points, referred to as fiber and matrix bonds, are shown in Fig. 4.

The critical parameters that define the failure of these bonds under tension and compression are $\left(s_{f t}, s_{m t}\right)$ and $\left(s_{f c}, s_{m c}\right)$, respectively, and can be determined based on the experimental measurements. At least four different uniaxial tension test cases of an unnotched laminate should be performed by considering different lay-up configurations. As shown in Fig. 5, these four lay-up configurations can be chosen as (25/0/75), (50/0/50), (75/0/25), and (100/

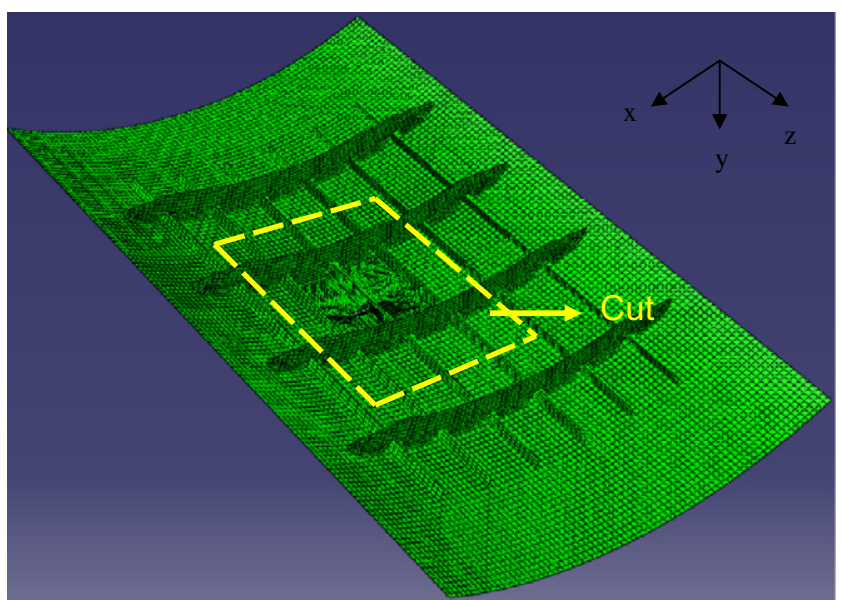

Fig. 13. Finite element discretization of the configured panel and cut boundaries for submodeling.
$0 / 0)$, where each value in the lay-up configuration indicates the percentage of $0^{\circ}, \pm 45^{\circ}$ and $90^{\circ}$ plies in the laminate, respectively. Tensile failure strain of the $(100 / 0 / 0)$ laminate corresponds to the critical stretch of a fiber bond under tension, $s_{f t}$. Linear extrapolation of the tensile failure strains for different lay-up configurations leads to the failure strain for the $(0 / 0 / 100)$ laminate, i.e., the critical stretch of a matrix bond under tension, $s_{m t}$.

The dominant failure mechanism for the fiber-reinforced composites under compression loading is microbuckling. Therefore, a simultaneous failure of fiber and matrix bonds can be assumed under compression loading. Hence, a uniaxial compression test for an unnotched quasi-isotropic laminate with a (25/50/25) lay-up can be performed to determine the critical stretch of both fiber and matrix bonds under compression, $s_{f c}$ and $s_{m c}$, respectively.

Interlayer damage represents the breakage of (interlayer) bonds between a layer and its adjacent layers above and below. Hence, it provides the extent of delamination between the adjacent layers. Therefore, the interlayer bonds are assumed to fail only in tension. The critical stretch value for the interlayer bonds, $s_{i t}$, can be obtained analytically by equating the energy consumed by an advancing Mode-I crack to the work required to break all interlayer bonds as

$s_{i t}=\sqrt{\frac{2 G_{I c}}{t E_{m}}}$

where $G_{I c}$ and $E_{3}$ correspond to the Mode-I critical energy release rate and elastic modulus of the matrix material, respectively, and $t$ is the ply thickness.

The transverse shear bonds can fail if the shear angle of the bonds exceeds the critical shear angle value, $\gamma_{c}$. This value can also

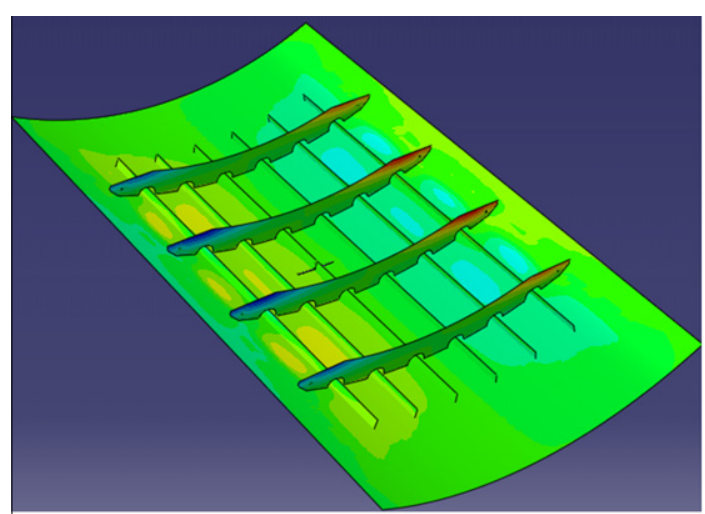

(a)

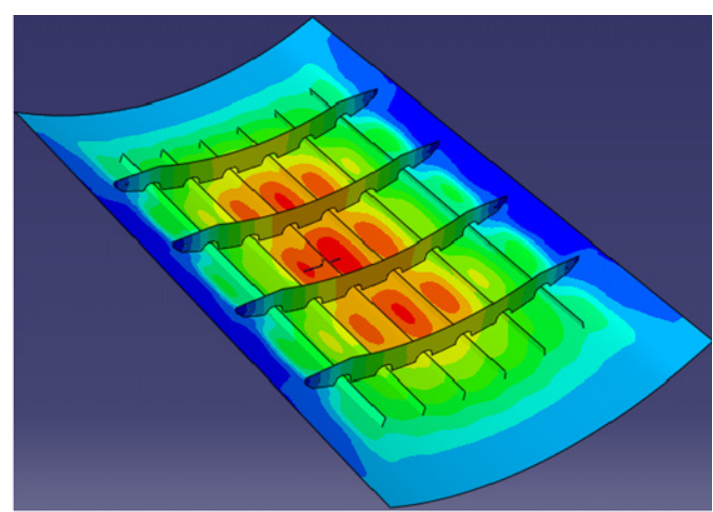

(b)

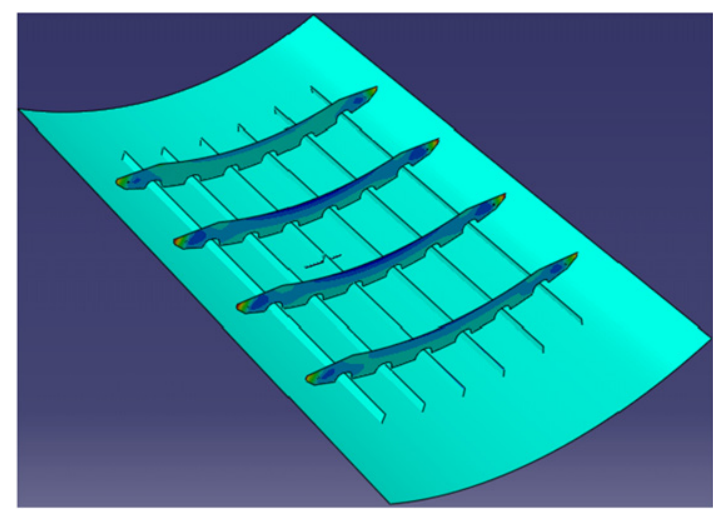

(c)

Fig. 14. Displacement variation due to internal pressure loading (a) $u$, (b) $v$, and (c) $w$. 
be obtained analytically by equating the energy consumed by an advancing Mode-II crack to the work required to break all shear bonds as

$\gamma_{c}=\sqrt{\frac{G_{I I c}}{t G_{m}}}$

where $G_{I I c}$ and $G_{m}$ correspond to the Mode-II critical energy release rate and the transverse shear modulus of the matrix material, respectively. The derivations of the relationships between the critical stretch value for the interlayer bonds, $s_{i n}$ and the Mode-I critical energy release rate and between the critical shear angle value, $\varphi_{c}$ and the Mode-II critical energy release rate, respectively, are given by Oterkus and Madenci [9].

\section{Coupled approach: Finite element method and peridynamic theory}

In order to achieve computational feasibility and robustness, the finite element method (FEM) and peridynamic theory are coupled through the submodeling approach. The global modeling is performed using the FEM while the PD theory is employed for submodeling in order to perform failure prediction. The PD analysis is performed by using the Emu code, Silling [10]. The primary assumption in submodeling is that the structural details of the submodel do not significantly affect the global model. Also, the boundaries of the submodel should be sufficiently far away from local features so that St. Venant's principle holds for a valid submodeling

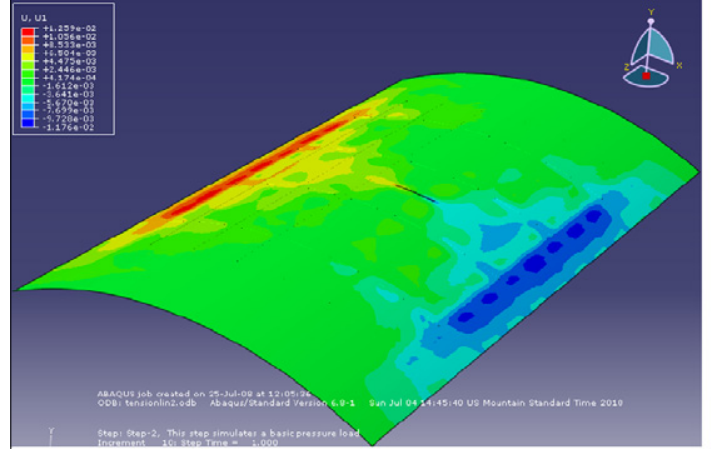

(a)

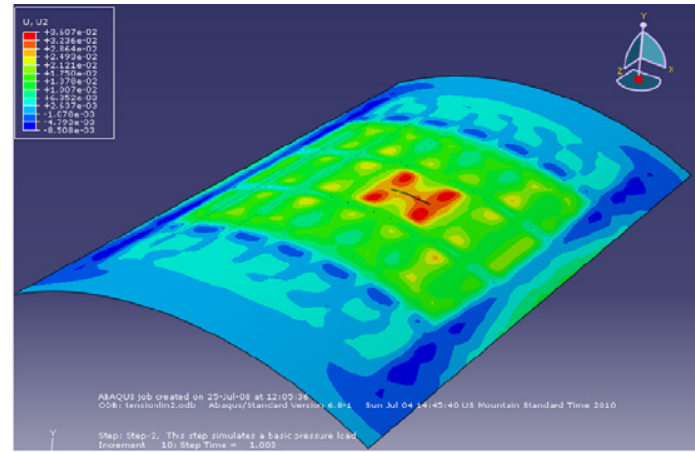

(b)

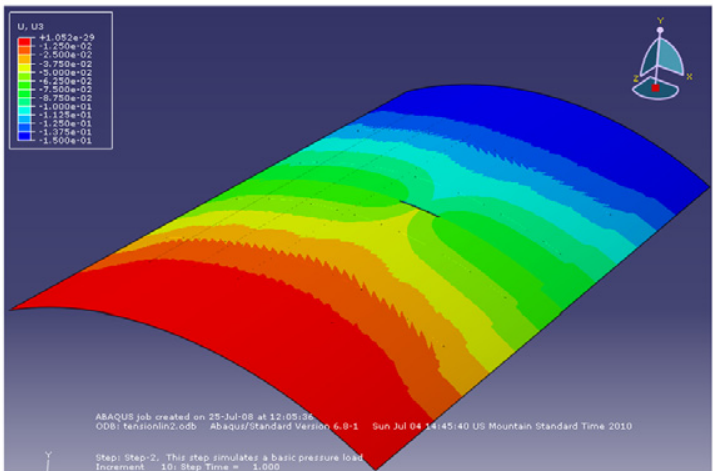

(c)

Fig. 15. Displacement variation due to axial tension loading (a) $u$, (b) $v$, and (c) $w$.

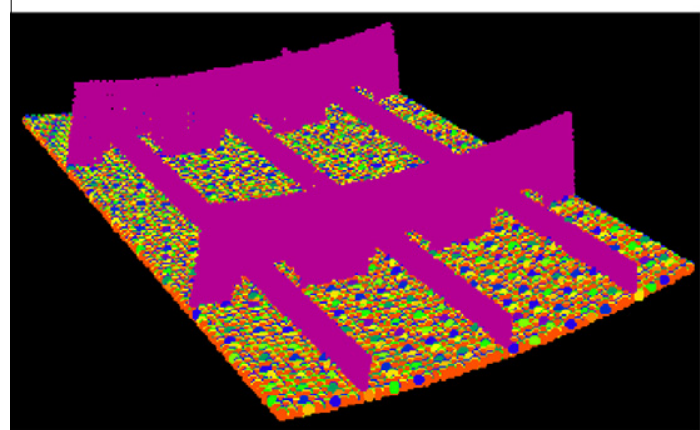

(a)

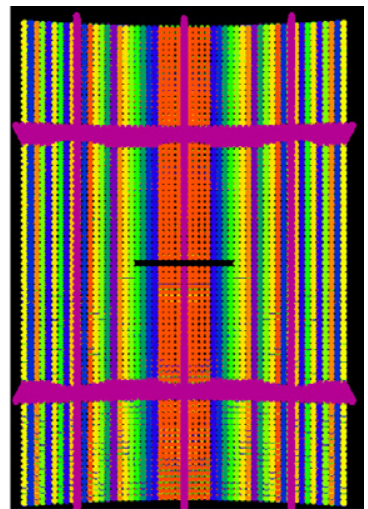

(b)

Fig. 16. Peridynamic analysis: (a) submodel and (b) top view of the submodel. 
analysis. The solution obtained from the global model along the boundary of the domain of interest is applied as displacement boundary conditions on the submodel. The global model should be refined enough to enable accurate calculation of the displacement on the boundary of the submodeling region. Also, different time discretizations of the displacement boundary condition

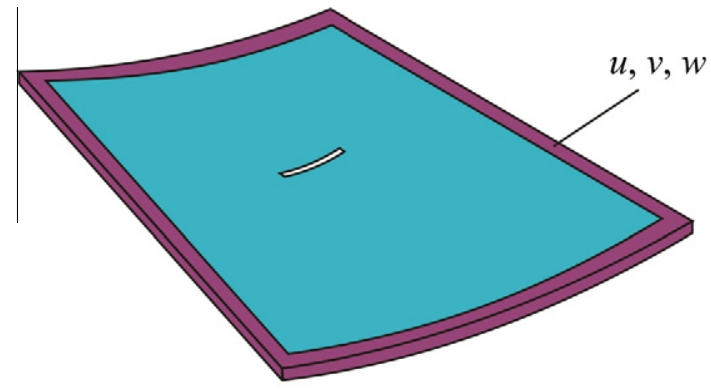

(a)

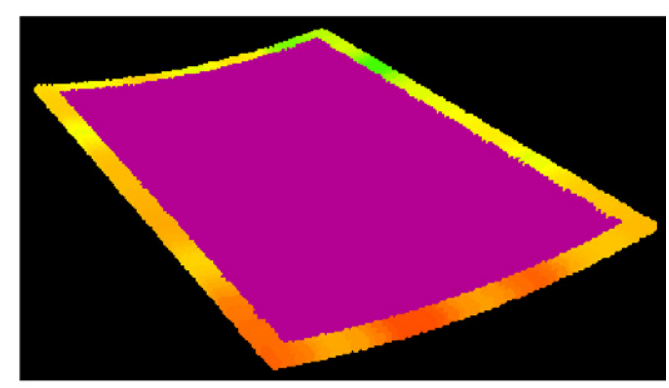

(b)

Fig. 17. Boundary domain in the panel skin: (a) in a representative figure and (b) in the actual peridynamic model.

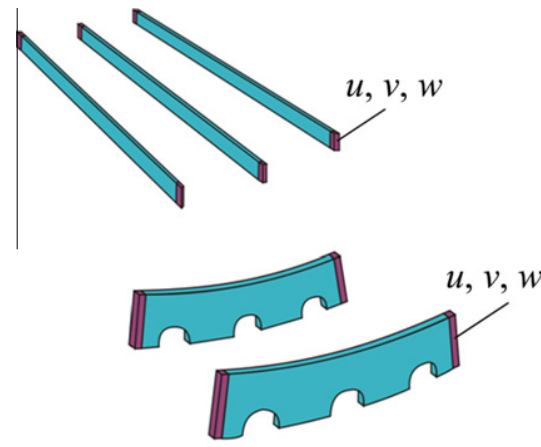

(a)

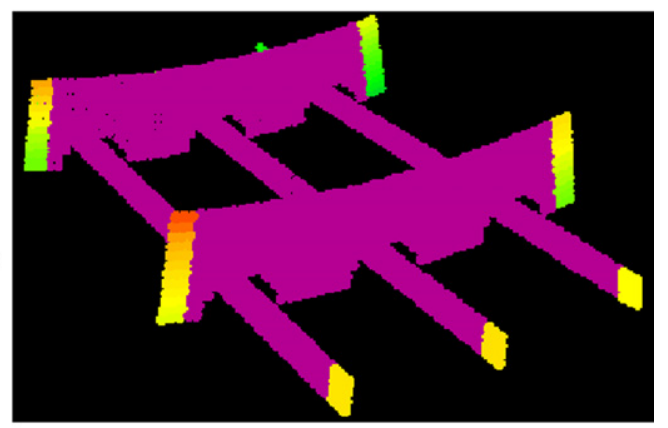

(b)

Fig. 18. Boundary domain in the stiffeners: (a) in a representative figure and (b) in the actual peridynamic model.

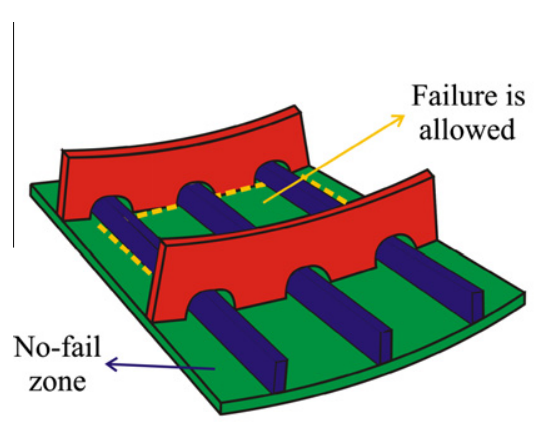

(a)

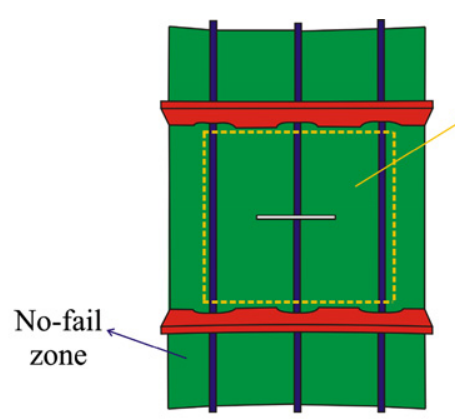

(b)

Fig. 19. Submodel: (a) no-fail zone representation and (b) top view of the submodel.
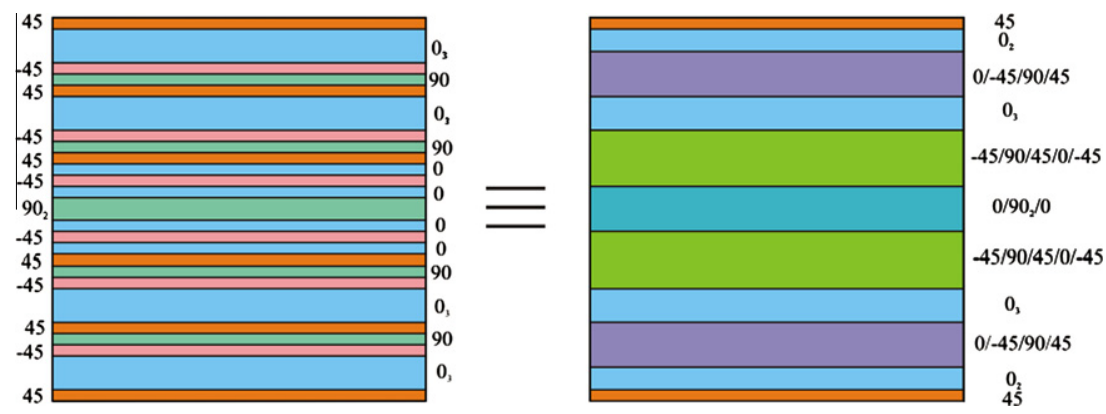

Fig. 20. Representation of a 34-ply panel skin by an equivalent 11-ply laminate. 
should be used because time-dependent fidelity of boundary conditions may affect results in submodeling.

The applicability of this approach is verified against finite element predictions by considering a cylindrical composite shell un-

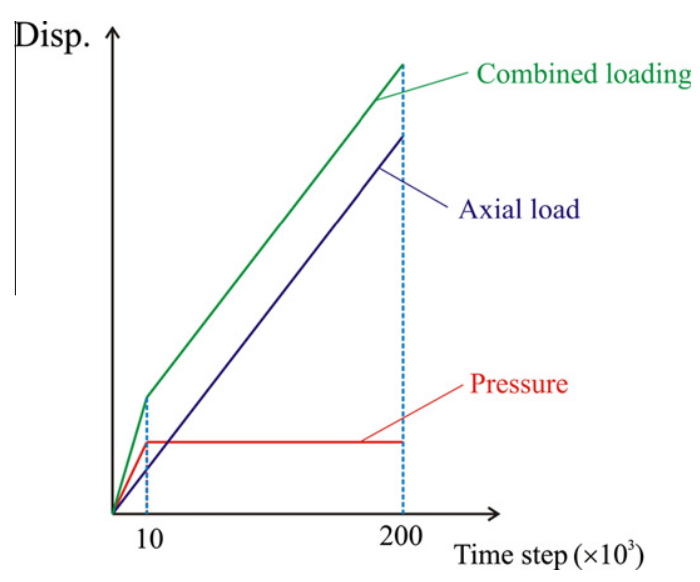

Fig. 21. Application of displacement constraints due to internal pressure and axial tension loading. der internal pressure in the absence of failure. A stiffened curved composite panel with a slot, referred to as NASA Panel 67, is also considered to compare initial and final failure load, as well as failure mode predictions, against the test results obtained at NASA LaRC. This panel is subjected to both the internal pressure and axial tension loading conditions.

\subsection{Cylindrical composite shell under internal pressure}

The shell is made of glass/epoxy with a lay-up of $\left[0^{\circ} / 90^{\circ} /\right.$ $\left.45^{\circ} / 90^{\circ} / 0^{\circ}\right]_{s}$. The elastic material properties are specified as $E_{1}=38.6 \times 10^{9} \mathrm{~Pa}, E_{2}=8.27 \times 10^{9} \mathrm{~Pa}, G_{12}=4.14 \times 10^{9} \mathrm{~Pa}$, and $v_{12}=$ 0.26. It is worth noting that these values for the in-plane shear modulus and Poisson's ratio deviate from their limiting values, $G_{12}=2.84 \times 10^{9} \mathrm{~Pa}$, and $v_{12}=0.33$, as dictated by Eq. (14). Fiber angle is specified with respect to $x$-axis as shown in Fig. 6 . The geometry of the shell is defined by the parameters $R=5 \mathrm{~m}, W=2 \mathrm{~m}$, $t=0.1 \mathrm{~m}$, and $\theta=23^{\circ}$. The shell is simply supported along the edges and subjected to an internal pressure of $P=1.0 \times 10^{4} \mathrm{~Pa}$, as shown in Fig. 6. The geometric dimensions constitute of a shallow shell; thus, the domain can be approximated in term of flat "square" subdomains in the numerical solution of the equations.

As shown in Fig. 7, the submodeling region is defined by the cut boundaries. The displacements and rotations obtained from the

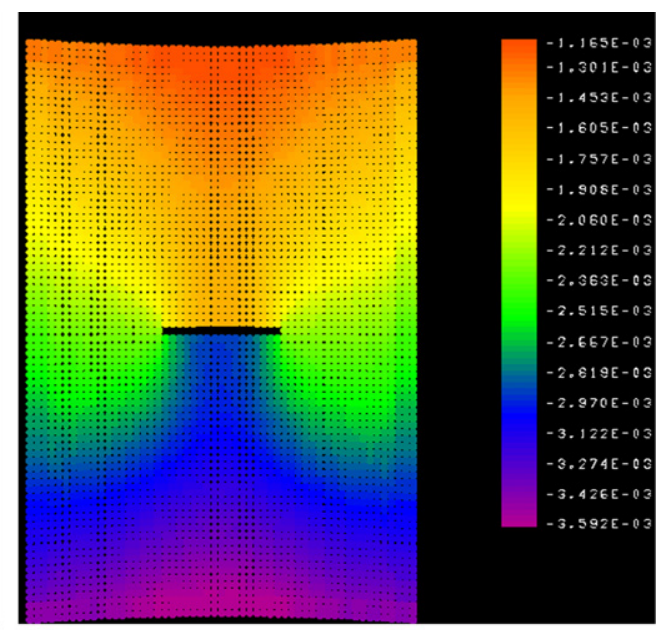

(b)

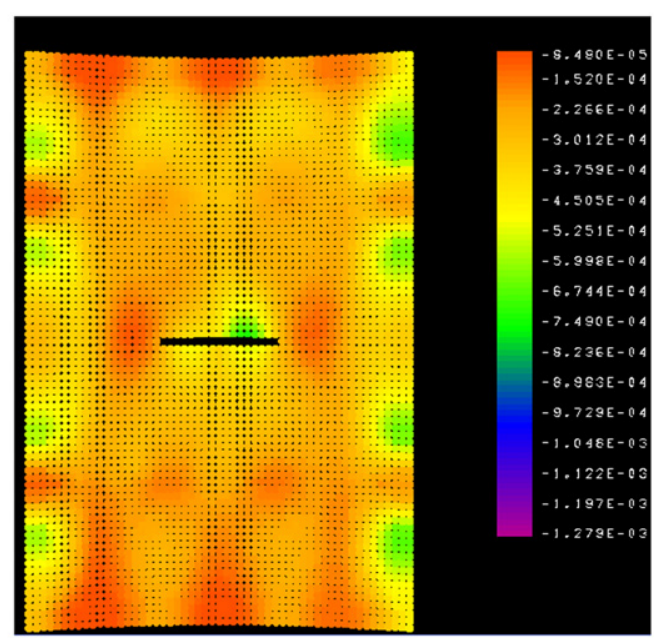

(c)

Fig. 22. Displacement field in the submodel just before initial failure occurs: (a) $u$, (b) $v$, and (c) $w$. 
global model (Figs. 8 and 9) along the cut boundaries serve as boundary conditions. They are interpolated at the material points located along the cut boundaries of the PD submodel. The steady-state solution is obtained by using adaptive dynamic relaxation [11].

Based on the mid-plane displacement components, $u_{0}(x, y)$, $v_{0}(x, y)$, and $w_{0}(x, y)$, and rotations, $\theta_{x}(x, y), \theta_{y}(x, y)$, and $\theta_{z}(x, y)$, the boundary displacements through the thickness of the cut boundaries are obtained based on the Kirchhoff kinematics from

$u(x, y, z)=u_{0}(x, y)-z\left(u_{0}(x, y) / R-\theta_{y}(x, y)\right)$

$v(x, y, z)=v_{0}(x, y)-z \theta_{x}(x, y)$

$w(x, y, z)=w_{0}(x, y)$

These boundary displacements are imposed in the PD model of the domain of interest as shown in Fig. 10. Comparison of the out-ofplane displacement predictions from the finite element and PD analyses in the submodeling region are in excellent agreement, as shown in Fig. 11.

\subsection{Failure prediction in a stiffened composite panel with a central slot}

\subsubsection{Finite element analysis of the entire panel}

The curved panel geometry and dimensions are shown in Fig. 12. The panel has six stringers and four frame-webs, with a central slot at the center of 4 th stringer. The global model of the stiffened curved composite panel shown in Fig. 13 is constructed by using the finite element method, utilizing a commercially available code, ABAQUS. The finite element analysis is performed by employing S4R5 type elements.

The panel skin is a 34-ply composite laminate with a lay-up sequence of $\left[45^{\circ} / 0^{\circ}{ }_{3} /-45^{\circ} / 90^{\circ} / 45^{\circ} / 0^{\circ}{ }_{3} /-45^{\circ} / 90^{\circ} / 45^{\circ} / 0^{\circ} /-45^{\circ} /\right.$ $\left.0^{\circ} / 90^{\circ}\right]_{s}$. The thickness of each ply is specified as $0.0055 \mathrm{in}$. The panel skin is made of IM-7/PETI-5, with material properties $E_{1}=21.9 \times 10^{6} \mathrm{psi}, E_{2}=1.21 \times 10^{6} \mathrm{psi}, v_{12}=1 / 3$, and $G_{12}=0.405 \times$ $10^{6} \mathrm{psi}$. The stringers are composed of 50 plies with a lay-up composition of $(48 / 32 / 20)$. The ply thickness of the stringers is the same as the one used in the panel skin. The composite frame-webs are made of a single-ply unidirectional lamina with a ply thickness of $0.124 \mathrm{in}$.

The panel is subjected to combined internal pressure and axial tension loading. However, the applied load is decomposed into two

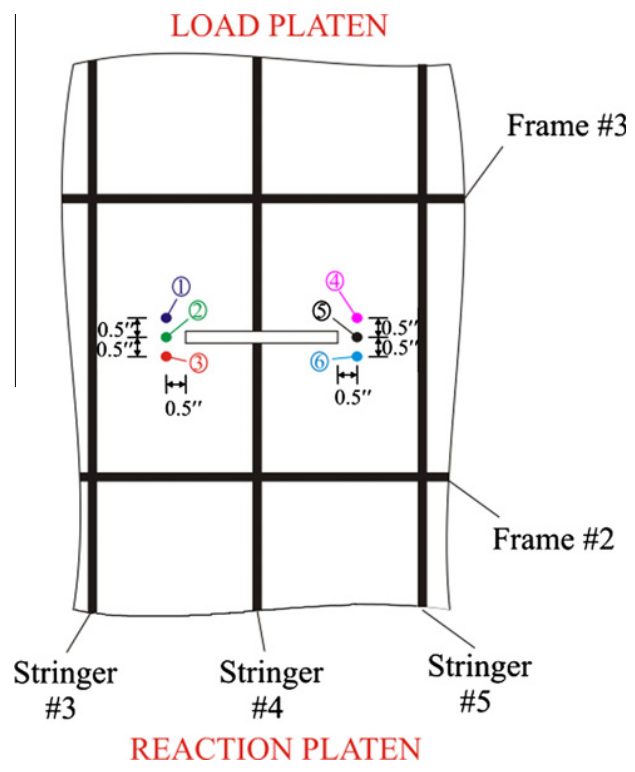

Fig. 23. Location of material points for monitoring displacement components. independent loading conditions of 7.2 psi internal pressure and uniaxial stretch of -0.75 in. in the global analysis with FEM. Uniax-
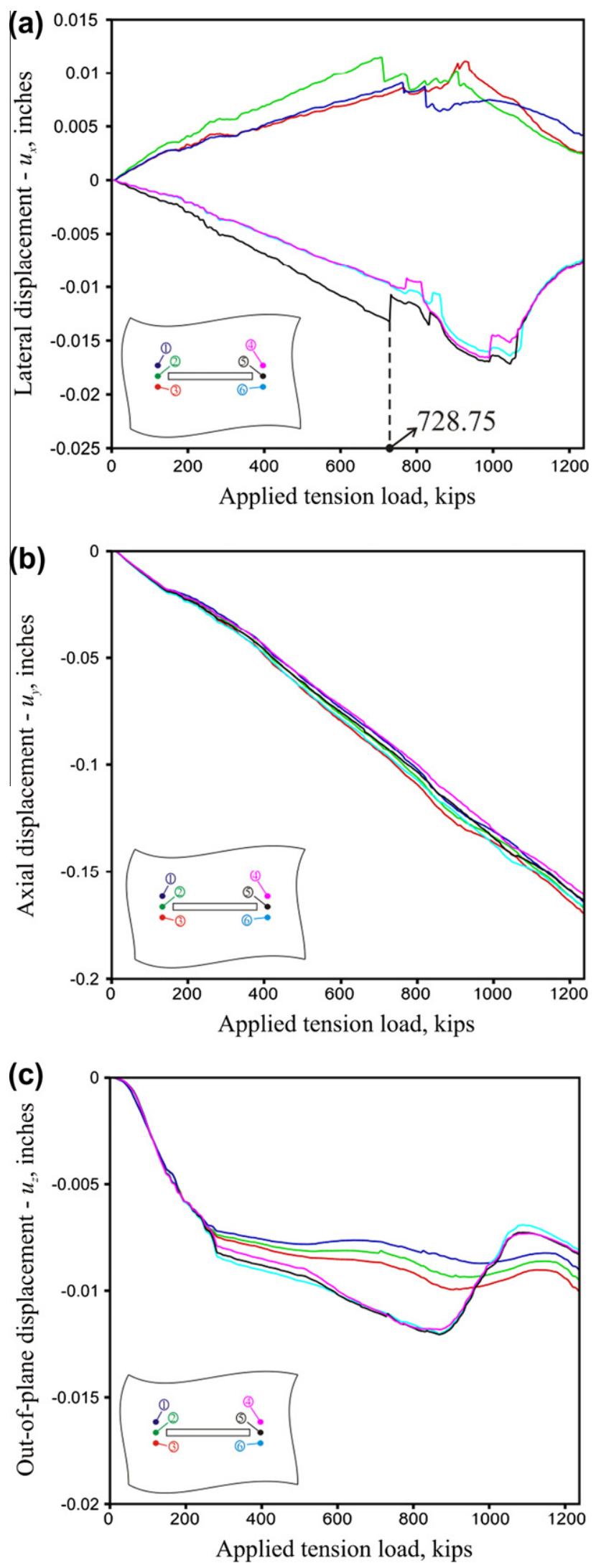

Fig. 24. Displacement variation of the monitored material points by time at the bottom ply: (a) lateral displacement, (b) axial displacement, and (c) out-of-plane displacement. 
ial tension loading is applied only along one horizontal edge of the panel while the displacements of the opposite horizontal edge are constrained. Arising from the applied internal pressure and uniaxial stretch, the displacement components in the entire stiffened panel are shown in the form of contour plots in Figs. 14 and 15, respectively. The displacement field obtained at several load steps provides the displacement boundary conditions along the cut boundaries of the submodel.

\subsubsection{Peridynamic analysis of the submodel}

The submodeling region includes three stringers and two frame-webs with a central slot, as shown in Fig. 16. The displacement boundary conditions in the peridynamic theory are applied to a volumetric region around the boundary of the domain, with a thickness approximately equal to the horizon size. The PD model is generated by using a single layer of material points per ply with a grid size of $\Delta x=0.5 \mathrm{in}$. The horizon radius is specified as $\delta=3.015 \Delta x$. Therefore, the displacement values from the global finite element model are extracted for the submodeling region and applied to the panel-skin and stiffener regions, as shown in Figs. 17 and 18 , respectively.

For the submodeling approach to be valid, the boundary region should not be affected by the local features inside the model. Therefore, it is necessary to define a no-fail zone where damage initiation is not allowed for the material points and damage cannot propagate further once it reaches the no-fail zone. In accordance with the St. Venant's principle, a large no-fail zone is chosen in the submodel region, which is shown in Fig. 19. The extent of the zone in which failure is allowed to occur consists of three of the stringers and ends very close to the frame-webs as shown in Fig. 19. The explicit size of this zone is specified as 18.11 in. by 18.11 in.

The panel skin is a 34-ply composite laminate with a lay-up sequence of $\left[45^{\circ} / 0^{\circ}{ }_{3} /-45^{\circ} / 90^{\circ} / 45^{\circ} / 0^{\circ}{ }_{3} /-45^{\circ} / 90^{\circ} / 45^{\circ} / 0^{\circ} /-45^{\circ} /\right.$ $\left.0^{\circ} / 90^{\circ}\right]_{s}$. Each layer of the laminate can be modeled by using the approach described in Section 3. However, inclusion of each of the 34 plies as an individual ply is computationally challenging. Therefore, the 34 plies are represented by an equivalent 11-ply laminate with the same laminate thickness, as shown in Fig. 20. The reduction of number of plies is achieved by considering sublaminates that have either quasi-isotropic or cross-ply layups.

The stringers are made of 50 plies with a lay-up composition of (48/32/20), and the composite frame-webs are made of unidirectional plies. In order to simplify the analysis, both the stringers and frame-webs are modeled as isotropic plates with the corresponding equivalent elastic modulus values. Their evaluated numerical values are close to each other; thus, the elastic modulus value is specified as $E_{e q v}=580.15 \times 10^{6} \mathrm{psi}$ for both stringers and

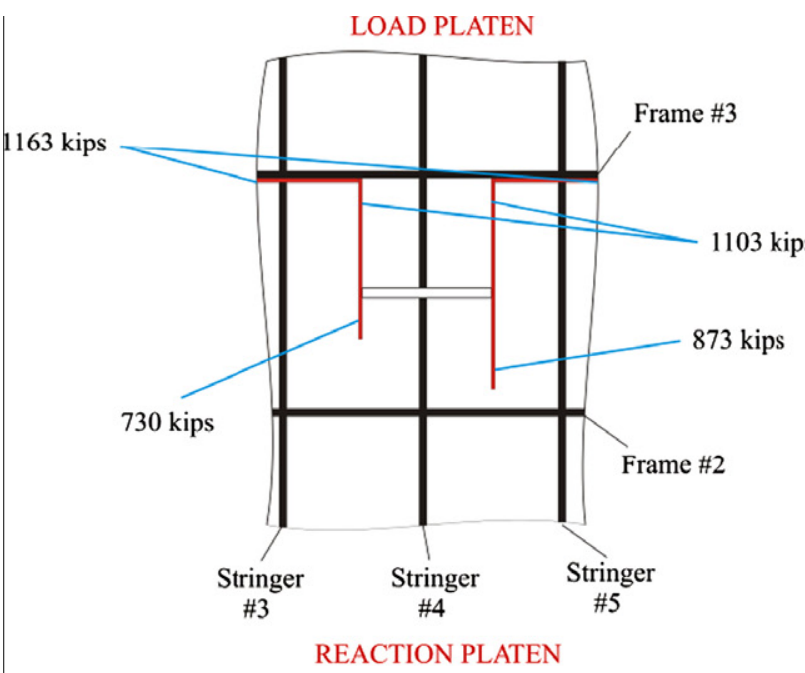

(a)

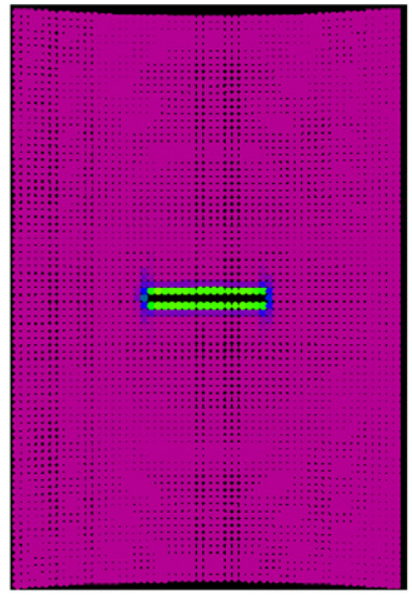

(b)

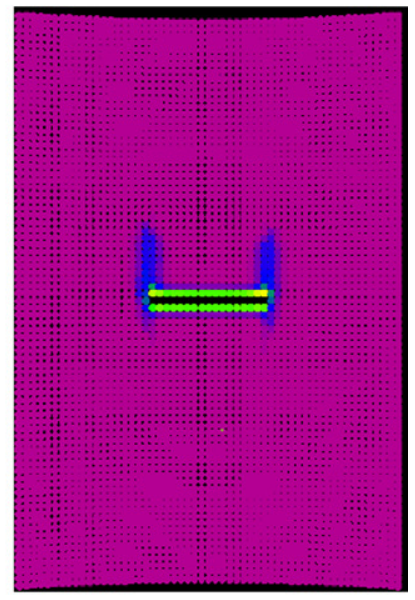

(c)

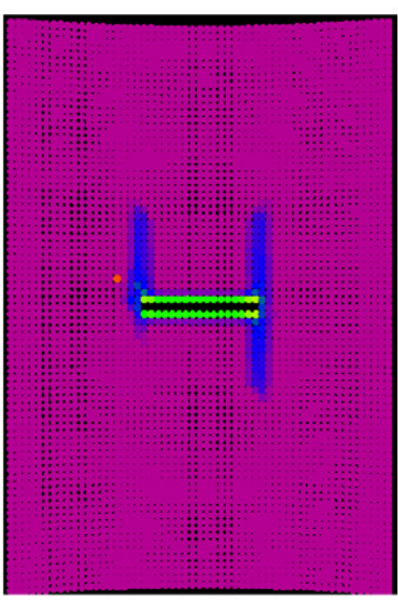

(d)

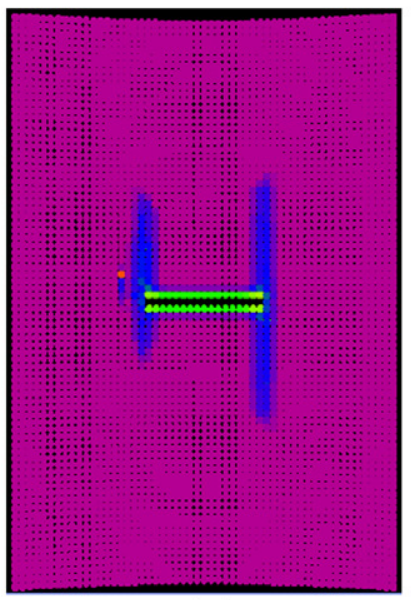

(e)

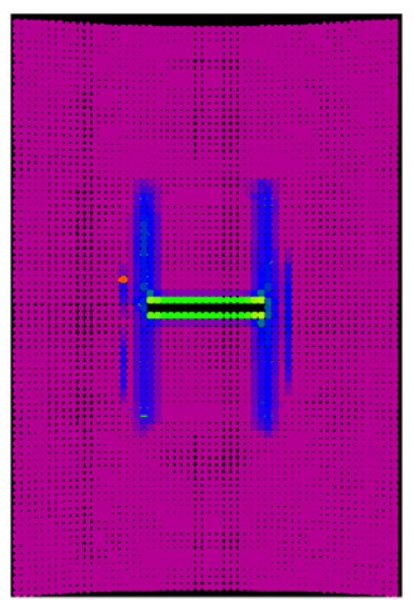

(f)

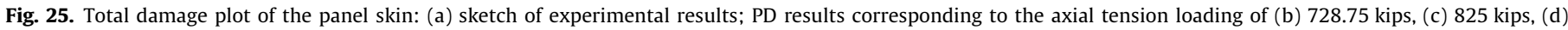
893.75 kips, (e) 962.5 kips, and (f) 1100 kips. 
frame-webs. Although this elastic modulus value is unrealistically high for an isotropic material, in this study, it only ensures the deformation response of a corresponding composite stringer and frame-web. The critical stretch parameters specified for the panel skin are $s_{f t}=0.008874, s_{m t}=0.004437, s_{f c}=s_{m c}=-0.00346, s_{i t}=$ 0.02 , and $\gamma_{c}=0.013$.

The displacement results extracted as boundary conditions for the submodeling region from both loading conditions are superposed as described in Fig. 21. In order to represent the test loading conditions, the steady-state condition for the internal pressure loading is obtained in a very short amount of time, i.e., 10,000 time steps. Therefore, the displacement constraints due to internal pressure loading are increased linearly for the first 10,000 time steps and then kept constant. In addition to the displacement boundary conditions in the submodel, internal pressure loading is applied as a body load. However, the displacement constraints due to the axial tension load have a constantly increased trend in order to determine the failure load of the panel. The total time steps required to reach the maximum applied load level were 200,000.

\section{Numerical results}

The contour variation of displacement components immediately before initial failure occurs are shown in Fig. 22. The PD analysis successfully captures the expected displacement variations due to the axial tension load, contraction due to Poisson's effect, and out-of plane deformation due to internal pressure and panel curvature.

The initial failure load is obtained by monitoring displacement components at six specific material points in each ply close to the right and left tips of the slot, as shown in Fig. 23. Hence, a total of 66 material points are monitored for 11 plies of the panel skin.

As shown in Fig. 24a, there is a sudden drop in the lateral displacements of material points 2 and 5 at an axial tension load of 728.75 kips. However, the axial displacements vary linearly for all time steps, without exhibiting any sudden drop (Fig. 24b). The out-of-plane displacements at applied load levels of 270 kips and 870 kips exhibit two kinks (Fig. 24c). These two points are not considered as indicative of failure initiation because the dominant loading is due to axial tension. Therefore, the presence of kinks on the lateral displacements is more crucial than the out-of-plane displacement.

After the initial failure occurs, a linear increase in the axial load is maintained until the crack reaches the no-fail zone. Experimental damage results and the damage evolution of the total damage plots from PD predictions are shown in Fig. 25a-f. As shown in Fig. 25a, two cracks at the left and right edges of the notch propagate in the downward direction and stop before they reach Frame \#2 at the load levels of 730 kips and 873 kips, respectively. However, the cracks that propagate in the upward direction reach Frame \#3 and move along the frame after changing their direction. The load level at which these cracks cross Stringers \#3 and \#5 is determined as 1163 kips.

According to the PD results, after the initial failure occurs (Fig. 25b), two cracks propagate in the upward direction from both sides of the crack (Fig. 25c). Then, another crack starts to propagate at the right tip of the slot (Fig. 25d), followed by fourth crack at the left tip (Fig. 25e). At the end of the applied load level, 1100 kips, a symmetric failure pattern occurred both at the left and right tips of the slot, as well as in the upward and downward part of the panel skin. The overall failure mode observed in PD simulations corresponds to a splitting mode and is similar to the test results (Fig. 25a). Furthermore, the experimental load level at which the cracks reach Frame \#3, i.e., 1103 kips, is very close to the failure load value when the crack reaches the no-fail zone boundary close to the stiffeners.

\section{Conclusions}

A methodology combining the FEM and PD theory has been introduced for realistic prediction of damage initiation and growth. This methodology is capable of assessing the durability of complex composite structures. It accounts for laminate thickness and laminate anisotropy (lay-up); it also separately addresses the material failure in the fiber, matrix, and interfaces between the fiber and matrix. The analysis involves the concept of submodeling; the global modeling is performed using the finite element method and the PD theory is employed for submodeling and failure prediction. Displacements from the global model are applied to the submodel as boundary conditions, and the initiation and propagation of damage are predicted based on the PD theory.

The simulations capture failure modes among each ply, which are usually distinct; they heavily depend on fiber direction, which is realistically exhibited in the current results. When compared against the experimental observations, it is observed that the current methodology is able to capture the correct failure behavior. The predicted values of the initial and final failure loads due to combined internal pressure and axial tension loadings are remarkably close to those measured during the test. Also, the splitting failure mode at the final damage state is similar to experimental observations.

\section{References}

[1] Silling SA. Reformulation of elasticity theory for discontinuities and long-range forces. J Mech Phys Solids 2000;48:175-209.

[2] Askari E, Xu J, Silling S. Peridynamic analysis of damage and failure in composites. In: 44th AIAA/ASME/ASCE/AHS/ASC aerospace sciences meeting and exhibit, Reno, NV, AIAA-2006-88; 2006.

[3] Colavito KW, Kilic B, Celik E, Madenci E, Askari E, Silling S. Effect of void content on stiffness and strength of composites by a peridynamic analysis and static indentation test. In: 48th AIAA/ASME/ASCE/AHS/ASC structures, structural dynamics, and materials conference, Honolulu, HI, AIAA-2007$2251 ; 2007$.

[4] Colavito KW, Kilic B, Celik E, Madenci E, Askari E, Silling S. Effect of nanoparticles on stiffness and impact strength of composites. In: 48th AIAA/ ASME/ASCE/AHS/ASC structures, structural dynamics, and materials conference, Honolulu, HI, AIAA-2007-2021; 2007.

[5] Xu J, Askari A, Weckner O, Razi H, Silling S. Damage and failure analysis of composite laminates under biaxial loads. In: 48th AIAA/ASME/ASCE/AHS/ASC structures, structural dynamics, and materials conference, Honolulu, HI, AIAA2007-2315; 2007.

[6] Kilic B, Agwai A, Madenci E. Peridynamic theory for progressive damage prediction in centre-cracked composite laminates. Compos Struct 2009;90: 141-51.

[7] Oterkus E, Barut I, Madenci E. Damage growth prediction from loaded composite fastener holes by using peridynamic theory. In 51st AIAA/ASME/ ASCE/AHS/ASC structures, structural dynamics, and materials conference, Orlando, FL, AIAA-2010-3026; 2010.

[8] Silling SA, Askari E. A meshfree method based on the peridynamic model of solid mechanics. Comput Struct 2005;83:526-1535.

[9] Oterkus E. Peridynamic theory for modeling three-dimensional damage growth in metallic and composite structures. PhD Thesis, University of Arizona; 2010.

[10] Silling SA. EMU code version emu 2.6.17. Sandia National Laboratories, Albuquerque; 2009.

[11] Kilic B. Peridynamic theory for progressive failure prediction in homogeneous and heterogeneous materials. PhD Thesis, University of Arizona; 2008. 